Ann. Biol. anim. Bioch. Biophys., I972, 12 (4), 647-659.

\title{
INFLUENCE DE LA SUPPLÉMENTATION DE LA RATION PAR DES DOSES CROISSANTES DE DL-MÉTHIONINE SUR LA TENEUR EN ACIDES AMINÉS LIBRES DU JAUNE D'CEUF
}

\author{
M. LARBIER et J. GUILLAUME \\ avec la collaboration technique de J.-P. Harscoat \\ Station de Recherches avicoles, \\ Centre de Recherches de Tours, I. N. R. A., \\ 37380 Nouzilly
}

\section{RÉSUMÉ}

\begin{abstract}
Des poules pondeuses âgées de 35 semaines au début de l'essai sont nourries pendant 6 semaines avec le même aliment complet $\mathrm{T}$ dont la source de protéines, constituées de maiss, tourteau d'arachide et farine de plumes hydrolysées, est additionnée de DL-méthionine et de L-lysine. Pendant une période expérimentale de 4 semaines, les animaux sont répartis en 5 lots et reçoivent des régimes qui ne diffèrent que par leur taux de méthionine. Celui-ci est variable et échelonné de 0,18 à 0,48 p. xoo. Enfin pendant 4 semaines (période post-expérimentale) tous les animaux sont de nouveau nourris avec l'aliment $T$.

Le poids de l'œuf est maximum lorsque l'aliment distribué contient un taux de méthionine supérieur ou égal à 0,28 p. roo.

La teneur du vitellus en aciđes aminés libres totaux diminue dans les lots qui reçoivent des régimes à taux insuffisants de méthionine $(0,18$ ou $0,23 \mathrm{p}$. I00). Cette diminution est d'autant plus rapide et durable que la déficience en cet acide est accentuée. A l'opposé, un excès a peu d'influence.

Mieux que la concentration en acides aminés libres totaux, la teneur du vitellus en méthionine + cystine libres semble refléter la richesse du régime en méthionine. Les variations ne sont signicafitivement corrélées au taux de méthionine dans l'aliment qu'au cours de la $4^{\mathbf{e}}$ semaine de la période expérimentale.

La méthionine et la cystine libres du vitellus n'ont pas la même cinétique de variation. La concentration de la première traduit dès la $3^{\mathrm{e}}$ semaine de la période expérimentale l'apport alimentaire de méthionine, tandis que les variations de la seconde sont plus tardives mais durables.
\end{abstract}

\section{INTRODUCTION}

La teneur en acides aminés du vitellus dépend de la composition azotée de l'aliment consommé par la poule pondeuse (BRAY et KELLY, I966). En particulier, un apport insuffisant de méthionine ou de lysine diminue la concentration de l'ensemble de ces constituants (LARBIER, Blum et GuILLAUME, I972).

La cinétique et les limites de ces variations peuvent être étudiées en comparant 
l'effet de rations renfermant des proportions variables d'un acide aminé essentiel : la méthionine qui constitue le plus souvent le facteur limitant dans les protéines utilisées dans l'alimentation de la Poule pondeuse. Aussi, avons-nous entrepris d'étudier son influence sur la concentration des acides aminés libres du vitellus lorsque son taux dans l'aliment varie de part et d'autre du besoin théorique.

\section{MATÉRIEL ET MÉTHODES}

$$
\text { I. - Animaux - Régimes }
$$

Les poules, issues d'un croisement commercial (M 519, SELAF, France) et âgées de 35 semaines sont élevées en cages individuelles. Elles sont nourries ad libitum et soumises à un éclairement de 16 heures par nycthémère.

Elles reçoivent pendant 6 semaines le même aliment témoin $(T)$ à base de maïs et de tourteau d'arachide, supplémenté en L-lysine $\mathrm{HCl}$ et $\mathrm{DL}$-méthionine $(0,25$ et o, Io p. roo respectivement) de manière à couvrir les besoins protéiques de la poule pondeuse (NATIONAL RESEARCH COUNcIL, I966). Cet aliment renferme en particulier 0,28 p. Ioo de méthionine et 0,28 p. Ioo de cystine.

On constitue ensuite 5 lots homogènes de 7 poules chacun sur le critère du poids de l'œuf. Pendant 4 semaines (période expérimentale), on distribue à 4 lots des régimes expérimentaux, le cinquième continuant d'être nourri avec l'aliment $T$. Les régimes qui sont tous issus de l'aliment de base ne diffèrent que par le taux de supplémentation en DL-méthionine, qui varie de o à 0,3 p. Ioo. Leur composition figure au tableau $\mathbf{r}$.

\section{TABLEAU I}

Composition centésimale des régimes alimentaires

\begin{tabular}{|c|c|c|c|c|c|c|}
\hline & Régime de base & Régime témoin & \multicolumn{4}{|c|}{ Autres régimes } \\
\hline Maîs . . . . . . . . . . . . & 50 & 50 & 50 & 50 & 50 & 50 \\
\hline Tourteau d'arachide ...... & 15 & 15 & 15 & 15 & 15 & 15 \\
\hline Farine de plumes hydrolysées & 3 & 3 & 3 & $\mathbf{3}$ & 3 & 3 \\
\hline Amidon $\ldots \ldots \ldots \ldots \ldots \ldots$ & 10 & 10 & 10 & 10 & 10 & 10 \\
\hline Huile de maïs $\ldots \ldots \ldots \ldots \ldots$ & $\mathbf{2}$ & 2 & 2 & 2 & 2 & 2 \\
\hline Saccharose $\ldots \ldots \ldots \ldots \ldots$ & 9,37 & 9,27 & 9,37 & 9,32 & 9,17 & 9,07 \\
\hline $\begin{array}{l}\text { Complément minéral } \\
\text { vitaminique }\left(^{1}\right) \ldots \ldots \ldots\end{array}$ & 10,38 & 10,38 & 10,38 & 10,38 & 10,38 & 10,38 \\
\hline L - lysine $\mathrm{HCl} \ldots \ldots \ldots \ldots \ldots$ & 0,25 & 0,25 & 0,25 & 0,25 & 0,25 & 0,25 \\
\hline DL - méthionine ......... & 0 & 0,10 & 0 & 0,05 & 0,20 & 0,30 \\
\hline
\end{tabular}

Caracteristiques du regime de base ( $\left.{ }^{2}\right)$
Taux protidique $(\mathrm{N} \times 6,25) \ldots \ldots$
Énergie métabolisable .........
14,4 p. 100

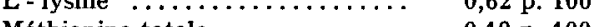
Méthionine totale ........... 0,18 p. 100
Acides aminés soufrés ......... 0,47 p. 100

(1) Apportant pour $100 \mathrm{~kg}$ d'aliment : Carbonate de calcium $7 \mathrm{~kg}$, Phosphate bicalcique $2 \mathrm{~kg}$, Chlorure de sodium $500 \mathrm{~g}$, Vitamine A $900000 \mathrm{UI}$, Vitamine $\mathrm{D}_{3} 100000 \mathrm{UI}, \alpha$ - tocophérol $2 \mathrm{~g}$, Vitamine $\mathrm{B}_{2} 0,25 \mathrm{~g}$, BHT $5 \mathrm{~g}$, Choline $100 \mathrm{~g}$, Vitamine $\mathrm{K}_{3} 0,2 \mathrm{~g}$, Pantothénate de calcium $0,2 \mathrm{~g}$, Vitamine $\mathrm{B}_{12} 0,3 \mathrm{mg}$, Vitamine PP $1,5 \mathrm{~g}$, Protoxyde vert de manganèse anhydre $12,6 \mathrm{~g}$, Oxyde de zinc anhydre $9,0 \mathrm{~g}$, Sulfate de fer $\mathrm{SO}_{4} \mathrm{Fe}, 7 \mathrm{H}_{2} \mathrm{O} 12,6 \mathrm{~g}$, Sulfate de cuivre $\mathrm{SO}_{4} \mathrm{Cu} 5, \mathrm{H}_{2} \mathrm{O} 0,99 \mathrm{~g}$, Carbonate de cobalt anhydre $0,054 \mathrm{~g}$, Iodure de potassium anhydre $0,189 \mathrm{~g}$, Gruau D 6,11 g, Carbonate de calcium 138,4 g, Avoine q.s.p. 10,380 kg.

(2) Déterminées par dosage sauf l'énergie métabolisable qui est calculée à l'aide des tables de l'A. E. C. (1972) (03 Commentry France). 
Pendant une $2^{\mathrm{e}}$ période également de 4 semaines (période post-expérimentale) tous les animaux sont de nouveau nourris avec l'aliment $T$.

\section{2. - Méthodes analytiques}

Les teneurs en acides aminés libres du vitellus sont déterminées sur des cufs fraîchement pondus, d'une part à la fin de la pré-expérience et d'autre part, les $3^{\mathrm{e}}$ et $4^{\mathrm{e}}$ semaines des périodes expérimentale et post-expérimentale. Pour chacune de ces semaines, on prélève au hasard deux œufs par poule et on réduit en deux échantillons les vitellus des œufs correspondant à l'ensemble des animaux d'un même lot.

Les jaunes d'œufs, débarrassés de leur membrane sont d'abord dilués dans un poids égal de sérum physiologique, L'extraction des acides aminés libres est obtenue à l'aide d'une solution d'acide picrique à I p. Ioo. Le traitement des extraits ainsi que le dosage des acides aminés libres sont effectués par chromatographie sur colonne de résine selon les techniques décrites par PAWLAK et PION (I968).

\section{RÉSULTATS}

\section{I. - Résultats zootechniques (tab1. 2)}

L'intensité de ponte (nombre d'œufs pondus par jour/nombre de poules) varie pour l'ensemble des animaux de 67,4 à $55,0 \mathrm{p}$. roo du début à la fin de l'essai. Les valeurs moyennes de chaque lot diffèrent peu. Compte tenu de la faiblesse des effectifs, de la brièveté de la période et de la variabilité intra-lots, il n'est pas possible d'en tirer de conclusion sur les effets de la supplémentation en méthionine.

La consommation journalière, tout aussi variable, passe de I30 $\mathrm{g}$ à I2I $\mathrm{g}$ par animal au cours de l'essai ; elle ne fournit pas non plus de renseignement significatif.

Le poids moyen de l'œuf évolue comme à l'ordinaire en sens inverse de l'intensité de ponte. Il est nettement influencé par l'apport de méthionine et pendant la période expérimentale. Il atteint une valeur maximale lorsque le taux de méthionine est égal ou supérieur à 0,28 p. 100 .

Malgré la distribution de l'aliment équilibré $T$ au cours de la période post-expérimentale, le poids de l'œuf reste diminué chez les animaux qui étaient antérieurement carencés en méthionine. Ainsi ce n'est pas seulement la composition de l'aliment du moment qui détermine le poids de l'œuf. Donc, une déficience provisoire en méthionine exerce sur le poids de l'œuf un effet dépressif dont la durée dépasse quatre semaines.

\section{2. - Teneur du vitellus en acides aminés libres totaux (tabl. 3)}

La teneur du vitellus en acides aminés libres totaux varie peu lorsque les poules sont exclusivement nourries avec l'aliment complet $T$. Les valeurs obtenues oscillent entre 339 et $345 \mathrm{mg} / \mathrm{Ioo} \mathrm{g}$ pendant la période pré-expérimentale pour l'ensemble des animaux et entre 340 et $350 \mathrm{mg} / 100 \mathrm{~g}$ au cours de l'essai proprement dit dans le lot témoin.

Elle diminue à la fois dans les lots qui reçoivent 0,18 et 0,23 p. Ioo de méthionine. Cet effet est d'autant plus rapide et durable que la carence en méthionine est accentuée. Avec l'aliment le plus déficient, la diminution a lieu dès la $3^{e}$ semaine de 


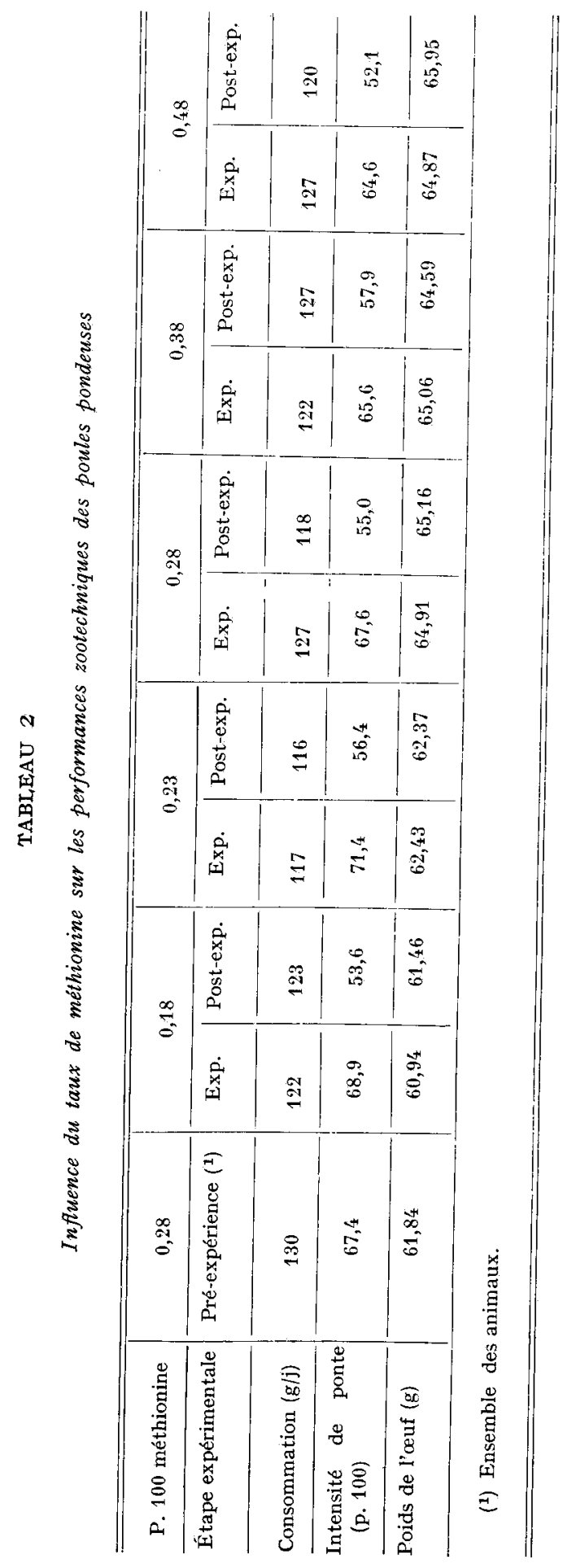


la période expérimentale. Dans le cas d'une déficience légère $(0,23$ p. Ioo de méthionine) l'effet dépressif est transitoire et de courte durée. I1 se manifeste à la fin de la période expérimentale et persiste pendant les trois premières semaines de la post. expérience.

TABLEAU 3

Teneur du vitellus en acides aminés libres totaux

(en $\mathrm{mg} / \mathrm{roo} \mathrm{g}$ )

\begin{tabular}{|c|c|c|c|c|c|c|}
\hline \multicolumn{2}{|c|}{$\begin{array}{l}\text { Taux de méthionine } \\
\text { dans l'aliment (p. 100) }\end{array}$} & 0,18 & 0,23 & $\begin{array}{c}0,28 \\
\text { Témoin }\end{array}$ & 0,38 & 0,48 \\
\hline \multicolumn{2}{|c|}{ Période pré-expérimentale $\left({ }^{1}\right)$} & 345 & 342 & 342 & 339 & 344 \\
\hline \multirow{2}{*}{$\begin{array}{c}\text { Période } \\
\text { expérimentale }\end{array}$} & $3^{\theta}$ semaine & 334 & 353 & 340 & 360 & 362 \\
\hline & $4^{e}$ semaine & 328 & 316 & 346 & 340 & 354 \\
\hline \multirow{2}{*}{$\begin{array}{l}\text { Période post- } \\
\text { expérimentale }\left({ }^{1}\right)\end{array}$} & $3^{e}$ semaine & 291 & 314 & 345 & 345 & 338 \\
\hline & $4^{\mathrm{e}}$ semaine & 298 & 333 & 350 & 327 & 322 \\
\hline
\end{tabular}

(1) Aliment contenant 0,28 p. 100 de méthionine pour tous les lots.

Les valeurs en chiffres italiques sont celles obtenues lorsque les poules consomment l'aliment $\mathrm{T}$.

A l'opposé, un apport excédentaire en méthionine seulement entraîne une légère augmentation au cours de la $3^{\mathbf{e}}$ semaine de la période expérimentale. Le retour au régime $\mathrm{T}$ au cours de la post-expérience provoque dans les lots qui recevaient un aliment renfermant 0,38 ou $0,4^{8}$ p. Ioo de méthionine une diminution de la teneur du vitellus en acides aminés libres. A la fin de l'essai, les valeurs obtenues y sont légèrement plus faibles que dans le lot témoin.

\section{3. - Teneur du vitellus en acides aminés soufrés libres}

Dans le lot témoin, la concentration en méthionine + cystine libres reste relativement constante. Elle oscille entre 8,8 et $9, \mathrm{I} \mathrm{mg} / \mathrm{Ioo} \mathrm{g}$ et représente de 2,6 à 2,7 p. roo de la teneur totale en acides aminés libres du vitellus.

Cette concentration est modifiée sous l'effet de la dose alimentaire d'acides aminés soufrés (fig. I). Ėn particulier, elle est nettement diminuée lorsque l'aliment renferme une proportion de méthionine inférieure à celle du régime témoin. A la $4^{\text {e }}$ semaine de la période expérimentale, les variations en fonction du taux de méthionine dans l'aliment deviennent linéaires. Il existe alors une relation hautement significative $(r=0,996 ; \mathrm{DL}=9)$ représentée par l'équation :

$$
y=14,865 x+4,522 \text {, }
$$

où : $y=$ teneur du vitellus en méthionine + cystine libres exprimée en $\mathrm{mg} /$ Ioo de vitellus

$x=$ taux de méthionine dans l'aliment en $p$. roo. 

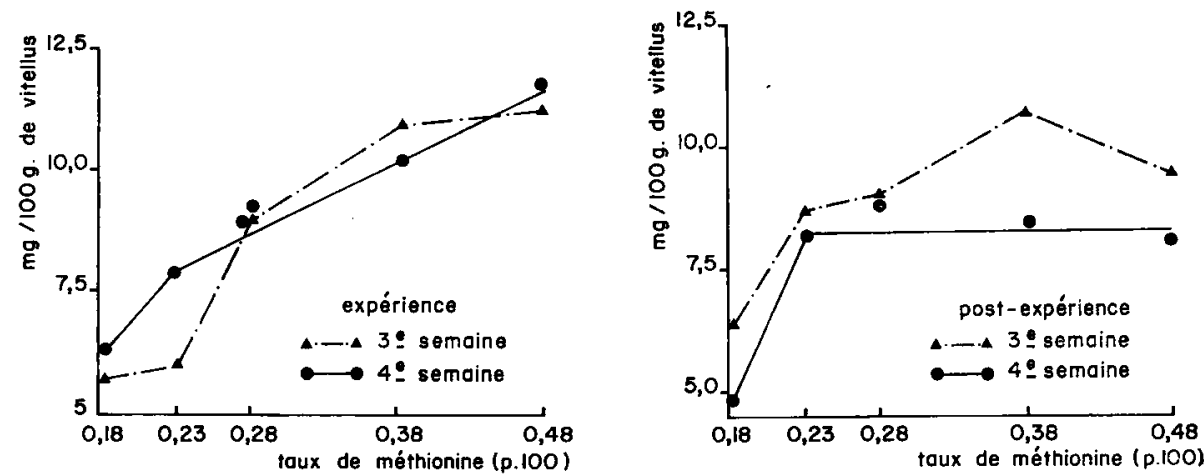

FIG. I. - Teneur du vitellus en méthionine + cystine libres en fonction du taux de mêthionine dans l'aliment
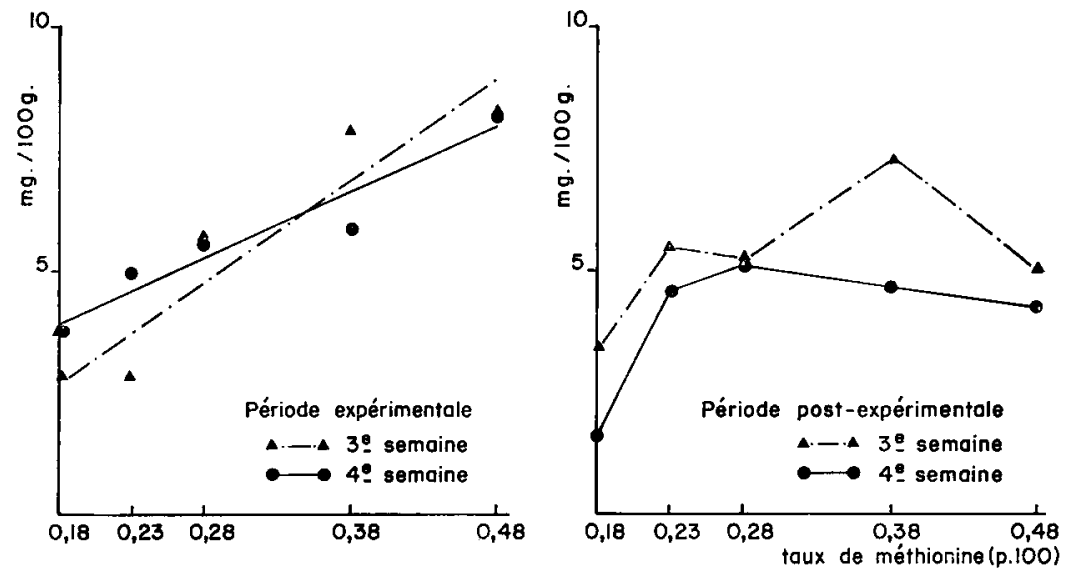

FIG. 2. - Teneur du vitellus en méthionine libre en fonction du taux de méthionine dans l'aliment
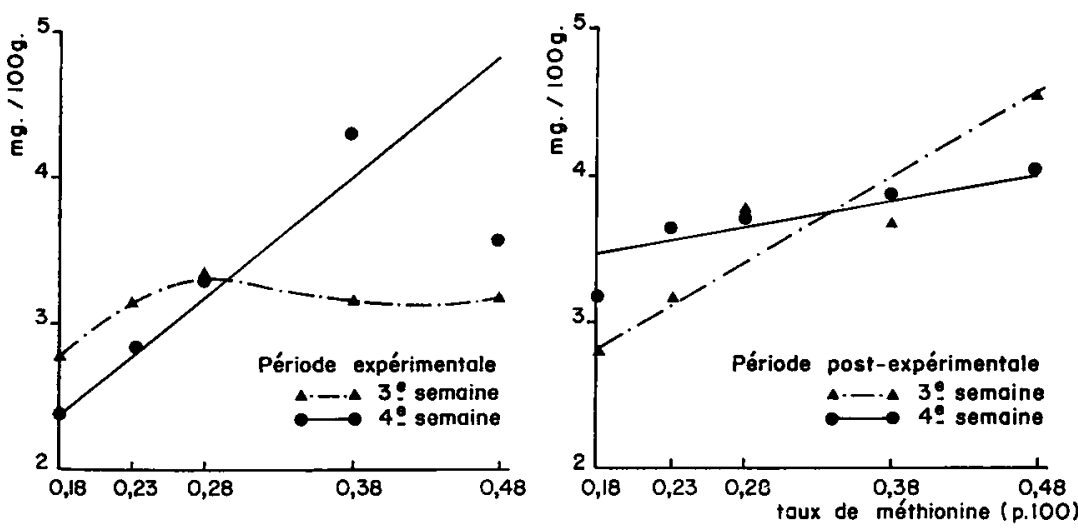

Fig. 3. - Teneur du vitellus en cystine libre en fonction du taux de méthionine dans l'aliment 
Le retour au régime témoin pendant la période post-expérimentale a des effets différents selon la composition de l'aliment distribué au cours de la période expérimentale (fig. 2). A la $3^{\mathrm{e}}$ semaine, on observe une diminution de la teneur en méthionine $(0,48 \mathrm{p}$. Ioo) et une légère augmentation pour le lot qui en contenait $0,23 \mathrm{p}$. Ioo. Les valeurs obtenues dans les autres lots ne sont pas modifiées. Par contre, à la $4^{\text {e }}$ semaine, seule le lot qui était le plus carencé en méthionine reste profondément affecté.

La méthionine et la cystine n'ont pas la même dynamique de variation au cours de l'essai. Pendant la période expérimentale, il existe toujours une relation linéaire significative entre la teneur du vitellus en méthionine libre et le taux de méthionine dans l'aliment ( $r=0,942$ et 0,954 respectivement à la $3^{\mathrm{e}}$ et la $4^{\mathrm{e}}$ semaine) (fig. 3). La corrélation n'est plus significative dès que l'ensemble des animaux est remis au régime équilibré T. Dans le cas de la cystine (fig. 4), les variations de teneurs, d'abord irrégulières, ne deviennent linéaires qu'à la $4^{\mathrm{e}}$ semaine de la période expérimentale, mais elles le demeurent jusqu'à la fin de l'essai.

\section{4. - Autres acides aminés libres (tab1. 4, 5 et 6 )}

Pour certains acides aminés libres, les concentrations dans le vitellus semblent varier parallèlement aux taux de supplémentation en DL- méthionine (tabl. 7). Il en est ainsi de la thréonine à la fin de la période expérimentale et au début de la post-expérience. Dans le cas des acides aspartique et glutamique, de l'alanine, de la leucine, de l'isoleucine, de la valine et de l'ornithine, la corrélation n'est généralement significative qu'à la $4^{\mathrm{e}}$ semaine de la période expérimentale.

Il apparait ainsi clairement que l'apport de méthionine ne modifie pas de façon identique la teneur du vitellus en acides aminés non soufrés. Dans la majorité des cas, la corrélation entre apport alimentaire en méthionine et teneur en acides aminés libres est positive et élevée à la fin de la période expérimentale. Elle le demeure dans une certaine mesure à la $3^{\mathrm{e}}$ semaine de la post-expérience. Ces acides aminés se comportent de façon voisine de la cystine. Cependant à la $4^{\mathrm{e}}$ semaine de la postexpérience, seule la concentration de celle-ci reste corrélée à l'apport antérieur de méthionine. 


\section{TABLEAU 4}

Évolution de la teneur en acides aminés libres du jaune d'ceuf avec le régime témoin (o, Io p. roo de méthionine ajoutée)

(mg/roo g)

\begin{tabular}{|c|c|c|c|c|c|c|c|c|}
\hline & \multicolumn{2}{|c|}{ (1) } & \multicolumn{2}{|c|}{ (2) } & \multicolumn{2}{|c|}{ (3) } & \multicolumn{2}{|c|}{$(4)$} \\
\hline & & p. 100 & & p. 100 & & p. 100 & & p. 100 \\
\hline Acide aspartique . & 220,9 & 6,2 & 22,0 & 6,4 & 20,8 & 6,0 & 21,2 & 6,0 \\
\hline Thréonine ....... & 23,3 & 6,9 & 22,1 & 6,4 & 24,2 & 7,0 & 21,6 & 6,2 \\
\hline Sérine ........ & 23,3 & 6,9 & 25,3 & 7,3 & 23,4 & 6,8 & 24,6 & 7,0 \\
\hline Acide glutamique. & 55,7 & 16,4 & 55,9 & 16,2 & 54,8 & 15,9 & 55,8 & 16,0 \\
\hline Proline $\ldots . . .$. & 17,2 & 5,1 & 18,0 & 5,2 & 19,2 & 5,6 & 18,2 & 5,2 \\
\hline Glycine......... & 7,8 & 2,3 & 8,0 & 2,3 & 8,1 & 2,4 & 8,2 & 2,3 \\
\hline Alanine ......... & 13,2 & 3,9 & 13,8 & 4,0 & 12,4 & 3,6 & 12,9 & 3,7 \\
\hline Valine.......... & 21,6 & 6,4 & 19,4 & 5,6 & 20,5 & 5,9 & 20,6 & 5,9 \\
\hline Cystine ........ & 3,4 & 1,0 & 3,4 & 1,0 & 3,8 & 1,1 & 3,7 & 1,1 \\
\hline Méthionine..... & 5,7 & 1,7 & 5,5 & 1,6 & 5,2 & 1,5 & 5,1 & 1,5 \\
\hline Isoleucine..... & 17,3 & 5,1 & 16,1 & 4,7 & 16,5 & 4,8 & 16,0 & 4,6 \\
\hline Leucine ........ & 31,7 & 9,3 & 30,5 & 8,8 & 31,3 & 9,1 & 31,6 & 9,0 \\
\hline Tyrosine......... & 23,3 & 6,9 & 21,6 & 6,2 & 28,8 & 8,4 & 28,0 & 8,0 \\
\hline Phénylalanine ... & 18,6 & 5,5 & 20,0 & 5,8 & 16,7 & 4,8 & 20,1 & 5,7 \\
\hline Ornithine $\ldots \ldots$ & 2,0 & 0,6 & 3,0 & 0,9 & 2,1 & 0,6 & 3,0 & 0,9 \\
\hline Lysine ......... & 28,7 & 8,4 & 30,7 & 8,9 & 29,2 & 8,5 & 29,6 & 8,5 \\
\hline Histidine ........ & 5,4 & 1,6 & 6,4 & 1,8 & 5,1 & 1,5 & 6,0 & 1,7 \\
\hline Arginine...$\ldots$. & 20,9 & 6,2 & 23,9 & 6,9 & 22,5 & 6,5 & 23,6 & 6,7 \\
\hline Total & 340,0 & & 345,6 & & 344,6 & & 349,8 & \\
\hline
\end{tabular}

(1) $3^{\mathrm{e}}$ semaine de la période expérimentale.

(2) $4^{\mathrm{e}}$

(3) $3^{\mathrm{e}}-\quad-$ post-expérimentale.

(') $4^{\mathrm{e}} \quad-\quad$ - $\quad$ - 


\section{TABLEAU 5}

Influence d'une carence partielle en méthionine sur la teneur en acides aminés libres du jaune d'ouf (mg/100 g)

\begin{tabular}{l|r|r|r|r|r|r|r}
\hline \hline & & & & & \\
D.L. méthionine \\
ajoutée (p. 100)
\end{tabular}

(1) $3^{\mathrm{e}}$ semaine de la période expérimentale.
(2) $4^{\mathrm{e}}$
- post-expérimentale.
(3) $3^{\mathrm{e}}$
(4) $4^{\mathrm{e}}-$ 


\section{TABLEAU 6}

Influence d'un excès de méthionine sur la teneur en acides aminés libres du jaune d'ouf (mg/roo g)

\begin{tabular}{|c|c|c|c|c|c|c|c|c|}
\hline \multirow{2}{*}{$\begin{array}{l}\text { D.L. méthionine } \\
\text { ajoutée (p. 100) }\end{array}$} & \multicolumn{2}{|c|}{0,20} & \multicolumn{2}{|c|}{0,10} & \multicolumn{2}{|c|}{0,30} & \multicolumn{2}{|c|}{0,10} \\
\hline & (1) & $(2)$ & (3) & (4) & (1) & (2) & (3) & (4) \\
\hline Acide aspartique . & 25,8 & 22,5 & 21,2 & 19,3 & 30,1 & 23,8 & 20,9 & 20,6 \\
\hline Thréonine ....... & 23,2 & 25,1 & 25,8 & 23,6 & 22,3 & 24,5 & 24,3 & 21,3 \\
\hline Sérine $\ldots . . . \ldots$ & 23,1 & 23,6 & 27,2 & 22,8 & 24,1 & 23,8 & 24,3 & 21,4 \\
\hline Acide glutamique . & 58,1 & 55,1 & 59,1 & 54,0 & 59,4 & 55,7 & 53,2 & 54,0 \\
\hline Proline....$\ldots$ & 14,3 & 17,5 & 18,3 & 17,7 & 17,4 & 16,3 & 17,6 & 16,7 \\
\hline Glycine.......... & 7,5 & 7,5 & 7,4 & 8,0 & 8,1 & 8,0 & 7,6 & 8,0 \\
\hline Alanine...... & 12,5 & 13,2 & 12,9 & 12,6 & 14,2 & 13,5 & 13,5 & 11,9 \\
\hline Valine.......... & 20,2 & 20,6 & 20,7 & 19,8 & 19,8 & 21,7 & 21,3 & 18,6 \\
\hline Cystine ........ & 3,1 & 4,3 & 3,6 & 3,8 & 3,1 & 3,5 & 4,6 & 4,1 \\
\hline Méthionine.... . & 7,8 & 5,8 & 7,2 & 4,6 & 8,2 & 8,2 & 5,1 & 4,0 \\
\hline Isoleucine $\ldots \ldots$ & 16,8 & 17,7 & 17,4 & 16,0 & 17,4 & 18,1 & 16,9 & 15,2 \\
\hline Leucine ........ & 30,0 & 31,1 & 30,0 & 30,3 & 31,6 & 32,3 & 29,9 & 29,9 \\
\hline Tyrosine........ & 25,5 & 21,7 & 21,9 & 22,8 & 25,5 & 24,5 & 23,4 & 21,9 \\
\hline Phénylalanine ... & 18,2 & 18,3 & 17,3 & 17,0 & 20,1 & 18,8 & 18,2 & 17,2 \\
\hline Ornithine $\ldots \ldots$ & 17,7 & 6,5 & 1,7 & 1,7 & 5,0 & 6,8 & 1,9 & 1,5 \\
\hline Lysine ......... & 28,6 & 27,2 & 29,0 & 25,4 & 29,6 & 30,2 & 27,9 & 28,5 \\
\hline Histidine ... & 4,8 & 5,3 & 4,7 & 5,0 & 5,7 & 5,3 & 5,1 & 5,6 \\
\hline Arginine $\ldots$ & 22,6 & 16,7 & 22,3 & 22,1 & 20,7 & 18,8 & 22,0 & 21,8 \\
\hline Total & 359,8 & 339,7 & $3 / 4,7$ & 326,5 & 362,3 & 353,8 & 337,7 & 322,2 \\
\hline
\end{tabular}

(1) $3^{\mathrm{e}}$ semaine de la période expérimentale.

(2) $4^{\mathrm{e}}$ -

(3) $3^{\mathrm{e}}-\quad$ - poxt-expérimentale.

(4) $4^{\mathrm{e}}$ 
ACIDES AMTNÉS LIBRES DU JAUNE D'CEUF

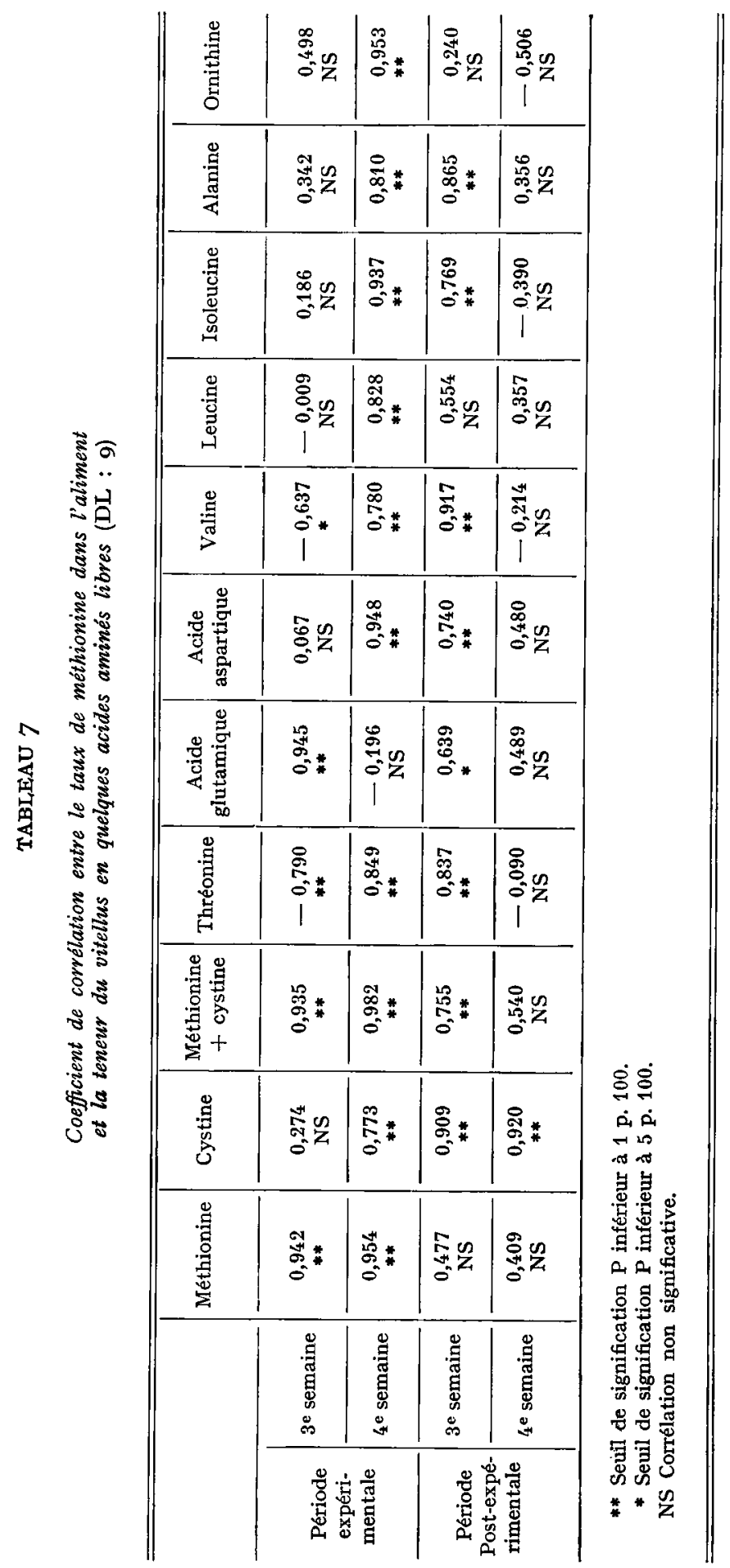




\section{DISCUSSION}

A la lumière des résultats concernant le poids de l'ouf, on vérifie que le taux de 0,28 p. Ioo de méthionine choisi pour l'aliment témoin est suffisant pour satisfaire les besoins de la poule M 5I9. Par ailleurs, compte tenu des mauvaises performances observées au cours de la post-expérience dans les lots qui étaient nourris avec un aliment à 0,18 ou 0,23 p. Ioo de méthionine, il ressort qu'une période de quatre semaines d'alimentation, avec un régime équilibré, est trop courte pour annuler les effets d'une carence antérieure en méthionine. Ein cela nous confirmons les résultats précédemment obtenus (LARBIER, Bi,um et GuILIAUME, I972.)

Les conditions expérimentales choisies dans notre essai permettent d'une part, de préciser les effets du taux alimentaire de méthionine (variant de $0, \mathrm{I} 8$ à 0,48 p. IOo) sur la concentration du vitellus en acides aminés libres et d'autre part, de suivre cet effet au cours du temps.

La relation entre méthionine alimentaire et ensemble des acides aminés libres du vitellus paraît en définitive résulter davantage d'une diminution de la teneur en méthionine dans le régime que de la teneur elle-même : l'effet du passage du régime excédentaire au régime témoin l'atteste. Il faut noter également que les variations quantitatives des acides aminés libres ont, comme les performances, un caractère durable lorsque l'aliment est nettement déficient en méthionine. Ici encore nous confirmons nos résultats antérieurs (LARBIER, BLUM et GUILLAUME, I97I). A l'opposé, les excès de méthionine ont peu d'effet.

Compte tenu de la durée de développement de l'ovocyte, tout se passe donc comme si l'influence d'un régime commençait à se manifester avant la phase de grand accroissement. Toutefois, cette influence prolongée d'une carence en méthionine peut s'expliquer par une action durable du régime sur le métabolisme protéique de la Poule et en particulier sur ses réserves azotées. La différence de cinétique de la méthionine et de la cystine résulterait de cette adaptation métabolique. Nous avons vu que la cystine libre ne traduit que tardivement le niveau d'acides aminés soufrés du régime. Tout se passe donc comme si une certaine quantité de cystine étant formée à partir de la méthionine, les réactions de synthèse nécessitaient un délai pour être activées ou ralenties. A cet égard, DANIEL, et WAISMAN, (I969) avaient montré que chez le Rat soumis à un excès ou à une déficience en méthionine, la concentration de méthionine libre dans le plasma est modifiée en même temps que l'activité de la cysthathionine synthétase, de la cystathionase et de la cystine sulfinate carboxylase et cela après quelques jours d'adaptation. De même, les variations quantitatives observées dans le cas des acides aminés libres non soufrés du vitellus résulteraient de l'effet de l'apport alimentaire de méthionine sur l'activité des enzymes du catabolisme (SANCHEZ et SWENDSEID, I969).

En définitive, ni la concentration totale en acides aminés ni celle en méthionine + cystine libres $d u$ vitellus ne reflètent avec certitude la composition azotée du régime ingéré au moment de l'analyse. Elles dépendent en effet du taux de méthionine contenu dans l'aliment antérieurement distribué à la Poule. 


\section{SUMMARY}

\section{EFFECT OF SUPPLEMENTING THE DIET WITH INCREASING AMOUNTS}

\section{OF DL-METHIONINE ON THE FREE AMINO ACID CON'TENT IN EGG YOLK}

Laying hens 35 weeks old at the start of the trial were fed for 6 weeks on the same complete diet, $T$, in which the sources of protein were maize, groundnut meal and hydrolyzed feathermeal and with DL-methionine and L-lysine added. For an experimental period of 4 weeks the birds were divided into 5 groups and given diets which differed in methionine, which ranged 0.18 to $0.4^{8}$ per cent. Then for a post-experimental period of 4 weeks diet $T$ was given to all birds.

Weight of egg was greatest when the feed given had 0.28 per cent methionine or more.

The free amino acid content of the yolk diminished in the groups given diets with insufficient methionine, o.I 8 or 0.23 per cent. The rate of decline and its duration were greater when the diet had less methionine. Excess methionine had little effect.

More than the concentration of total free amino acids, the content of free methionine + cystine in the yolk seemed to reflect the methionine content of the diet. The variations were only significantly correlated to the methionine content of the feed during the fourth week of the experimental period.

Free methionine and cystine in the yolk did not change in the same way. The concentration of methionine was affected by the supply of methionine in the feed from the 3 rd week of the experimental period, while changes in lysine were later but lasted longer.

\section{RÉFÉRENCES BIBIIOGRAPHIQUES}

Bray D. J., Kelly M., I.966. The effect of dietary lysine upon the free lysine content of egg yolk. Poult. Sci., 45, I425-I426.

Daniel R. G., Warsman H. A., r969. Adaptation of the weanling rat to diets containing excess methionine. J. Nutr., 99, 299-306.

Larbier M., Blum J.-C., Guillaume J., i972. Effets d'une déficience alimentaire en lysine et méthionine sur les performances de ponte et sur la teneur en acides aminés libres du jaune d'œuf. Ann. Biol. anim. Bioch. Biophys. (12 (1), 125-138).

National Research Council, r966. Nutrient requirements of poultry. Publication, I345.

Pawlak M., PIon R., I968. Influence de la supplémentation de proteines de blé par des doses croissantes de L-lysine sur la teneur en acides aminés libres du sang et du muscle du Rat en croissance. Ann. Biol. anim. Bioch. Biophys., 8, 517-530.

Sanchez A., Swendseid M. E., r969. Amino-acid levels and enzyme activity in tissues of rat force fed diets differing in methionine content. $J . N u t r ., 99, \mathrm{I}_{45}-\mathrm{I} 5 \mathrm{I}$. 